\title{
Single glycerol/water microdroplets standing on a superhydrophobic surface: Optical microcavities promising original applications
}

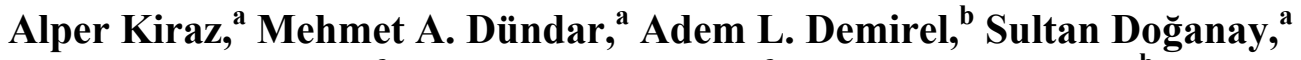 \\ Adnan Kurt, ${ }^{a}$ Alphan Sennaroglu, ${ }^{a}$ and Mehdi Y. Yüce ${ }^{b}$ \\ ${ }^{a}$ Department of Physics, Koç University, Sariyer, 34450 Istanbul, Turkey \\ ${ }^{b}$ Department of Chemistry, Koç University, Sariyer, 34450 Istanbul, Turkey
}

\begin{abstract}
Glycerol/water microdroplets take almost spherical shapes when standing on a superhydrophobic surface. Hence they are suitable to function as optical microcavities. Using Rhodamine B doped water microdroplets, large spectral tunability of the whispering gallery modes (WGMs) $(>5 \mathrm{~nm}$ ) was observed. Tunability was achieved by evaporation/condensation in a current controlled mini humidity chamber. Experiments revealed a mechanism stabilizing the volume of these microdroplets with femtoliter resolution. The mechanism relied on the interplay between the condensation rate that was kept constant and the size dependent laser induced heating. The radii of individual water microdroplets $(>5 \mu \mathrm{m})$ stayed within a few nanometers during long time periods (several minutes). By blocking the laser excitation for $500 \mathrm{msec}$, the stable volume of individual microdroplets were changed stepwise. Laser emission was also observed from Rhodamine B doped glycerol/water microdroplets using a pulsed, frequency-doubled Nd:YAG laser $(\lambda=532 \mathrm{~nm})$ as the excitation source. The observed largely tunable WGMs and laser emission can pave way for novel applications in optical communication systems. Besides due to the sensitivity of the WGMs to the size and shape of the microdroplets, the results can find applications in characterizing superhydrophobic surfaces and investigating liquid-solid surfaces.
\end{abstract}

Keywords: micro-optics, aerosols, surfaces, dye lasers, laser spectroscopy, fluorescence spectrsoscopy, droplet, superhydrophobic surface, whispering gallery mode.

\section{INTRODUCTION}

Microdroplets of hydrophilic liquids take almost spherical shapes when standing on a superhydrophobic surface. With their approximately truncated microsphere geometry, these microdroplets can provide a three dimensional confinement for light, specifically in the equatorial plane, parallel to the substrate. Provided a large contact angle, the whispering gallery modes (WGMs) of these optical microcavities [1] can possess relatively high quality factors. Besides, no additional position control technique such as electrodynamic trapping [2], or optical tweezing [3] is necessary to analyze the optical properties of these microdroplets.

The robustness of the experimental configuration and the relatively high quality of the observed WGMs make these microdroplets attractive for applications in optical communication systems and cavity quantum electrodynamics. Here we review our recent work in which single Rhodamine B doped glycerol/water microdroplets are used to demonstrate: (i) large spectral tunability [4], (ii) a volume stabilization mechanism [5], and (iii) laser emission [6]. We also comment on potential applications of the presented techniques. 


\section{EXPERIMENTAL}

\subsection{Superhydrohpobic surface preparation and microdroplet generation}

Superhydrophobic surfaces were prepared by spin coating a $20-60 \mathrm{mg} / \mathrm{ml}$ ethanol dispersion of hydrophobically coated silica nanoparticles on cover glasses at $2000 \mathrm{rpm}$ for 1 minute [7]. Hydrophobically coated silica nanoparticles (Degussa AG Aeroxide LE1 or LE2) had an average particle size of $7 \mathrm{~nm}$. Resulting superhydrophobic surfaces had nanometer scale surface roughness and were transparent to visible light. The average contact angle of millimetric water droplets on the superhydrophobic surface was measured to be $>150^{\circ}$. Prepared substrates were post-baked in an oven at $70{ }^{\circ} \mathrm{C}$ for one hour, for evaporation of the remaining ethanol.

A water solution doped with $20-50 \mu \mathrm{M}$ Rhodamine B, or 90/10 water/glycerol solution doped with $225 \mu \mathrm{M}$ Rhodamine B was sprayed onto a superhydrophobic surface using an ultrasonic nebulizer. In the experiments performed on microdroplets containing no glycerol, an electrically controlled mini humidity chamber was used. Joule heating of a $3 \Omega$ nichrome wire resistor was used to control the ambient humidity level of the chamber, causing evaporation or condensation in microdroplets. Heating currents between 0.15 and $0.3 \mathrm{~A}$ were used to obtain stable or nearly stable microdroplets.

In contrast, a humidity chamber was not used in the experiments perfomed on water/glycerol microdroplets. These microdropelts were kept in contact with the ambient atmosphere after their generation. Once sprayed, they quickly evaporated, and reached their equilibrium sizes. Due to the uncertainty in the amount of evaporation during the formation of the microdroplets, it was not possible to exactly determine the concentration of the Rhodamine B dye. The estimated concentrations were in the $0.225-2.25 \mathrm{mM}$ range.
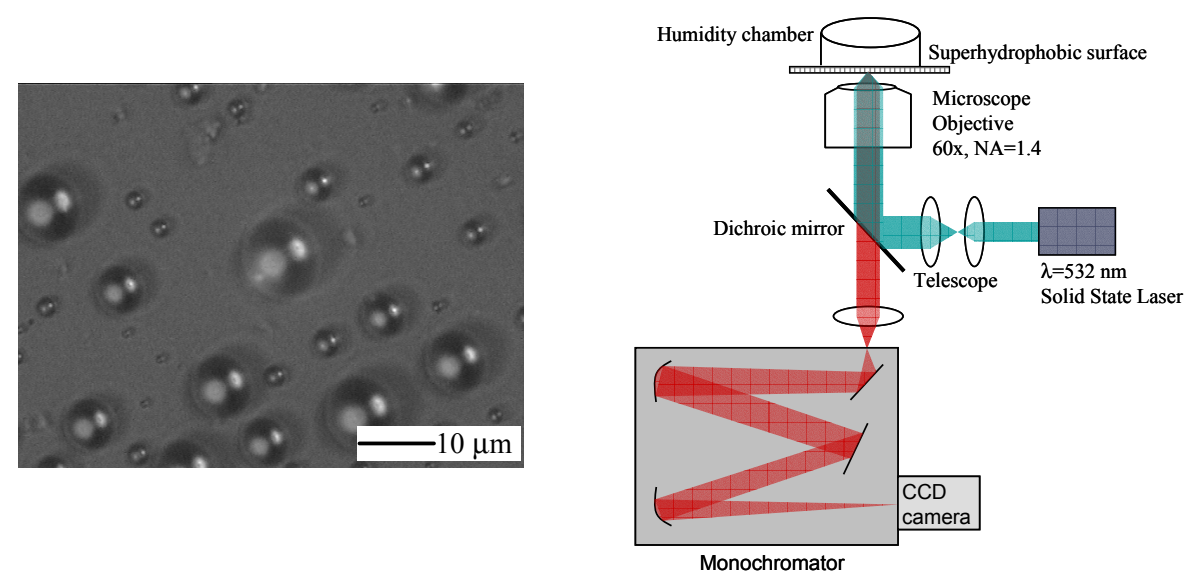

Fig. 1. (a) Top view of water microdroplets on a superhydrophobic surface. (b) Experimental setup.

\subsection{Experimental setup}

A continous wave (Sections 3.1 and 3.2) or pulsed (Section 3.3, pulsewidth=100 ns, repetation rate $=1 \mathrm{kHz})$ solid state green laser $(\lambda=532 \mathrm{~nm})$ was used to excite the microdroplets. The continuous wave laser was focused to a resolution limited spot near the outer surface while the pulsed laser was focused to a diameter of $12 \mu \mathrm{m}$. Excitation of the microdroplets and the collection of the emission were done by a high numerical aperture oil immersion microscope objective (NA=1.4, 60x). After being transmitted through a dichroic 
mirror (Q570LP), and a 1.5x magnifier element, the collected fluorescence was dispersed using a $50 \mathrm{~cm}$ monochromator and detected by a charge coupled device camera. With the selected 300 grooves $/ \mathrm{mm}$ grating and input slit width of $30 \mu \mathrm{m}$, a spectral resolution of 0.24 $\mathrm{nm}$ was achieved.

\section{RESULTS}

\subsection{Observation of largely tunable whispering gallery modes}

Optical microcavities [1] promise important applications in optical communication systems based on wavelength division multiplexing as optical switches and add/drop filters. Various demonstrations have been performed using semiconductor or polymer microdisk and microring microcavities [8]. However, despite the inherent potential, microcavity based devices have not found wide use in modern optoelectronic networks. One of the main obstacles has been tunability. Refractive index control of the constituent materials in a solid microcavity by changing the temperature $[9,10]$ or the electric field applied to liquid crystal cladding layers [11] enabled limited tunability within a maximum spectral range of $0.25 \mathrm{~nm}$.

Size and shape control provides an alternative mechanism for tuning microcavity resonances. Using solid optical microcavities a maximum tunability of $\sim 1 \mathrm{~nm}$ was demonstrated under a large external force [12]. In contrast, a liquid microcavity provides relatively easy control of its shape due to its viscous nature. An applied external force, electric field [13], or control of the evaporation/condensation kinetics can induce size and shape changes in liquids. We show that evaporation/condensation kinetics can be used to largely tune the WGMs of single water microdroplets resting on a superhydrophobic surface.

(a)

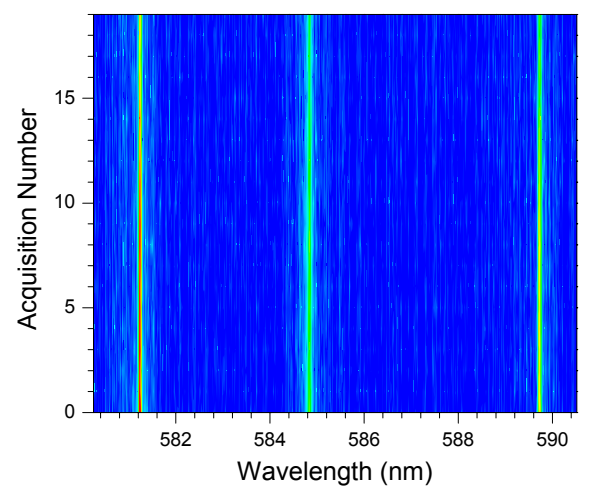

(b)

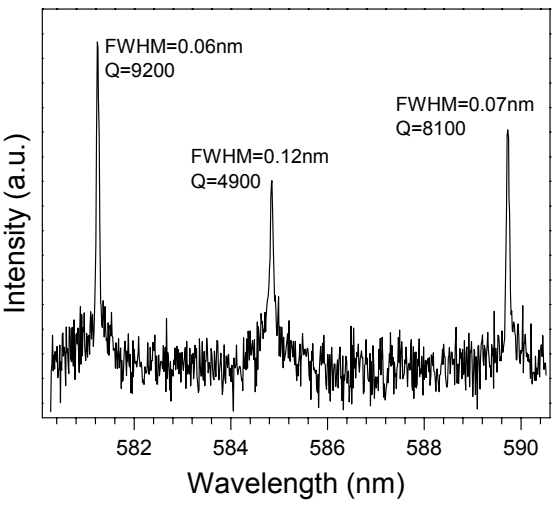

Fig. 2. (a) Contour plot of the emission spectra taken from a $6 \mu \mathrm{m}$ diameter microdroplet showing stable WGMs. Intensity values in arbitrary units increase from blue to red. Exposure and readout time of the camera are $2 \mathrm{sec}$ and $2.1 \mathrm{sec}$ respectively. (b) Sum of the 20 acquisitions in (a).

Figure 2 shows high resolution spectra obtained from a sizewise stable microdroplet. The temporal stability of the microcavity is demonstrated in the contour plot (Fig. 2a), and in the integrated spectrum (Fig. 2b). During 82.8 seconds of data acquisition, no spectral drift was observed in the WGMs within the resolution of the spectrometer setup. This corresponds to a size stability of $\sim 1 \mathrm{~nm}$. The mechanism giving rise to such high volume stabilization is explained in Section 3.2. The decrease in the intensity of the WGMs between consecutive acquisitions was due to photobleaching of Rhodamine B molecules. In Fig. 2 as well as in all 
other spectra shown in this article, we refrained ourselves from identifying the mode numbers and orders of WGMs, due to the errors caused by the nonspherical microdroplet geometry. In the low pumping regime where stimulated emission is negligible as compared to spontaneous emission, the quality factors (Q-factors) of the WGMs are given by the the full width at half maxima (FWHM) of the observed peaks. This condition was satisfied in our experiments. In Fig. 2b, a Q-factor of 4900 was observed for the peak at $584.84 \mathrm{~nm}$ while the Q-factors of the two other WGMs were determined to be larger than 8000 , which is almost the resolution limit of our experimental setup. Considering the microdroplet radius of $6 \mu \mathrm{m}$, the free spectral range (FSR) of the WGMs is expected to be larger than $8.5 \mathrm{~nm}$ [14] for an ideal water microsphere, yielding a minimum finesse (FSR/FWHM) of $\sim 120$ for the high quality WGMs.

WGMs were tuned in the directions of both evaporation and condensation as a function of the ambient humidity. Figure 3 shows the consecutive spectra taken from three different microdroplets. Evaporation was observed in Fig. 3a leading to a total spectral drift of $6.5 \mathrm{~nm}$, whereas in Fig. $3 \mathrm{~b}$ condensation caused a total spectral drift of $3.5 \mathrm{~nm}$ in the WGMs. In Fig. $3 \mathrm{c}$, the direction of evaporation was reversed by sudden increase of local humidity. After a spectral drift by $0.8 \mathrm{~nm}$ in the direction of evaporation, a spectral drift of $0.7 \mathrm{~nm}$ was observed in the direction of condensation.

(a)

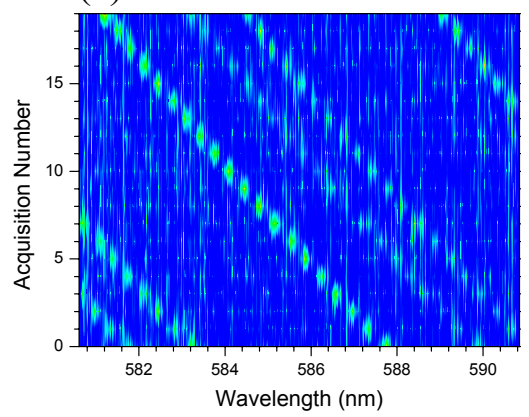

(b)

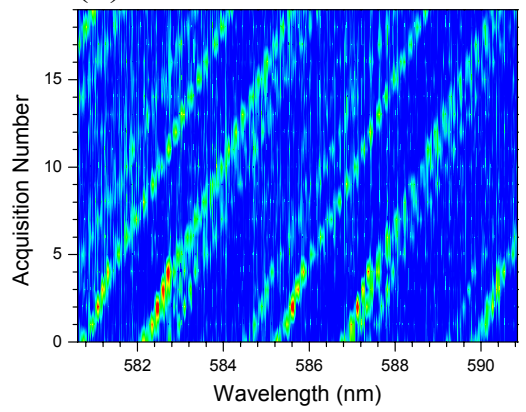

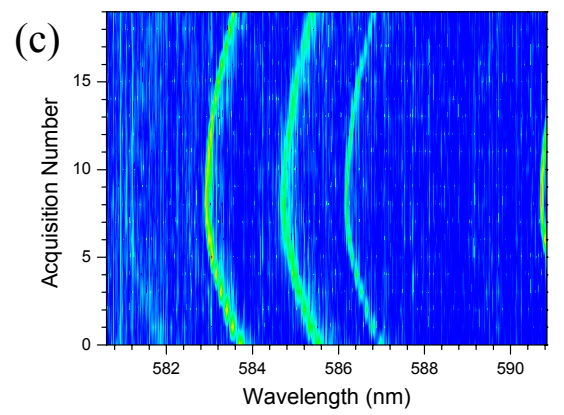

Fig. 3. Consecutive spectra taken from three microdroplets exhibiting (a) evaporation, (b) condensation, and (c) evaporation followed by condensation. The radii of the microdroplets were $6 \mu \mathrm{m}, 11 \mu \mathrm{m}$, and $6 \mu \mathrm{m}$ in (a), (b), and (c), respectively. The intensity values in arbitrary units increase from blue to red. Exposure and readout times of the camera are $2 \mathrm{sec}$ and $2.1 \mathrm{sec}$ in (a), (b), and (c).

\subsection{Observation of a volume stabilization mechanism}

Size control has been an important challenge in applications using microdroplets of liquids with relatively high vapor pressures. Only recently single water microdroplets suspended by optical tweezers were analyzed for long time periods $[3,15]$. In these experiments, volume 
stabilization was achieved by evaporation of initially salty microdroplets (concentrations $0.04-1.28 \mathrm{M}$ ) in a humidity controlled chamber.

In Section 3.1 we have demonstrated the stability as well as tunability of WGMs of Rhodamine B doped water microdroplets on superhydrophobic surfaces in a large spectral window. In this section, we show that the observed volume stabilization is due to the ambient humidity and size dependent laser absorption, and we introduce a mechanism that allows the volume of microdroplets to be changed stepwise.

Scattering of a plane electromagnetic wave by a dielectric sphere is explained by Lorenz Mie theory. Derivation of Lorenz - Mie theory has been extended to incorporate an incident focused Gaussian beam [16,17]. Enhanced internal field intensities have been predicted from these analyses when resonance conditions (spectral, spatial, and polarization) are met between the excitation laser beam and specific WGMs. The resulting enhanced absorption was demonstrated using Rhodamine 6G doped ethanol microdroplets in air [18]. Microdroplets having a critical size showed much larger absorption efficiencies compared to microdroplets with diameters differing by $4 \mathrm{~nm}$.

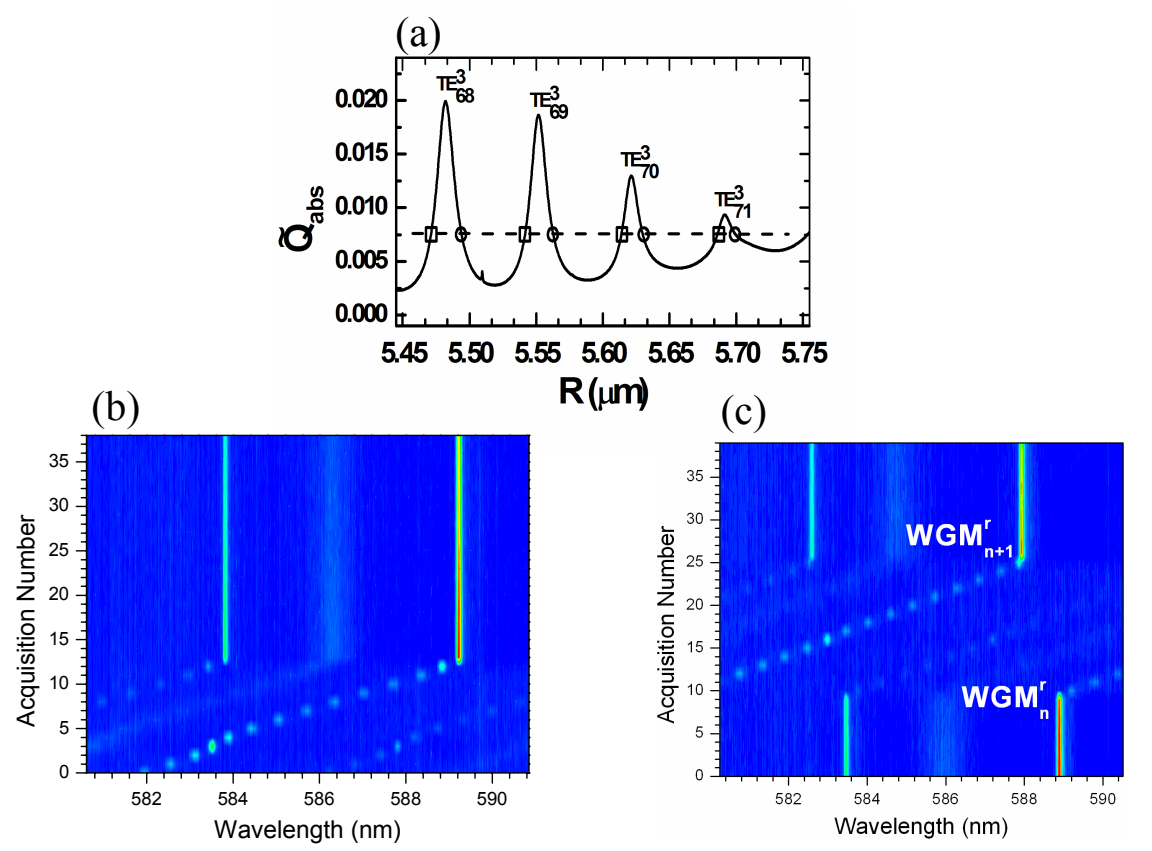

Fig. 4. (a) Calculated modified absorption efficiency as a function of radius (R) for a sphere having refractive index, equal to that of $50 \mu \mathrm{M}$ Rhodamine B doped water $\left(n=1.33+4 \cdot 10^{-5} \mathrm{i}\right)$. Focus is positioned away from the center of the sphere at a distance of $5.8 \mu \mathrm{m}$. Contour plots of emission spectra taken from single microdroplets showing (b) volume stabilization at acquisition 13 (c) volume stabilization at consecutive stable equilibrium points. The radii of the microdroplets were $6 \mu \mathrm{m}$ in (b) and (c). Exposure and readout times of the camera are $1 \mathrm{sec}$ and $2.1 \mathrm{sec}$ in (a), and (b).

Size dependent absorption phenomenon plays a crucial role in the realization of the reported high precision volume stabilization mechanism. Fig. 4a shows the calculated modified absorption efficiency $\left(\widetilde{Q}_{a b s}\right)$ as a function of radius $(R)$ for a sphere having a refractive index equal to that of $50 \mu \mathrm{M}$ Rhodamine B-doped water $\left(n=1.33+4 \cdot 10^{-5} i\right)$. This calculation simulates our experiment, with a tightly focused Gaussian beam $\left(\lambda=532 \mathrm{~nm}, \omega_{0}\right.$ $=250 \mathrm{~nm}$, linearly polarized along the $\mathrm{x}$ direction), and were calculated using the localized 
model developed by Gouesbet et al. [19] with an algorithm introduced by Lock [20]. $\widetilde{Q}_{a b s}$ is the ratio of the total power absorbed by the sphere to the power incident upon the projected area of the sphere and to the total power of the incident beam respectively. In Fig. 4a the first order modes are suppressed due to the high absorption coefficient. In Fig. 4a the focal point is located at $5800 \mathrm{~nm}$ (off axis illumination). In this figure only the TE modes are excited as a result of the polarization direction.

The size of the microdroplet is determined by the balance between the condensation rate $\left(\Gamma_{\text {cond }}\right)$, and the evaporation rate $\left(\Gamma_{\text {evap }}\right)$. In our experiments, $\Gamma_{\text {cond }}$ was kept at a constant value as indicated by the dashed line in Fig. 4a. Because the self-control stability mechanism was dominant near a resonance, the factors affecting evaporation kinetics other than the laser induced heating stayed nearly constant, achieving steady state conditions. Furthermore, within the range of the experimental parameters, we have observed an almost linear relationship between the laser induced heating and $\Gamma_{\text {evap }}$. Thus, in our experiments $\Gamma_{\text {evap }}$ was almost proportional to $\widetilde{Q}_{a b s}\left(\Gamma_{\text {evap }} \propto I \widetilde{Q}_{a b s}, I\right.$ is the laser intensity) and changed with the size of microdroplet as it shrunk $\left(\Gamma_{\text {cond }}<\Gamma_{\text {evap }}\right)$ or grew $\left(\Gamma_{\text {cond }}>\Gamma_{\text {evap }}\right)$ in size. For each WGM, the microdroplet can be at equilibrium at two different sizes as indicated by a solid square on the left of the peak and a solid circle on the right. The square corresponds to a stable equilibrium point where a self-locking mechanism is in effect: an increase in size due to condensation or a decrease in size due to evaporation is counterbalanced by a corresponding increase or decrease, respectively, in $\widetilde{Q}_{a b s}$ maintaining the equality. On the contrary, the circle corresponds to an unstable equilibrium: an increase in size causes $\widetilde{Q}_{a b s}$ to decrease and the microdroplet continues growing in size. Similarly, a decrease in size causes $\widetilde{Q}_{a b s}$ to increase and the microdroplet continues shrinking in size.

Fig. 4b shows the contour plots of consecutive fluorescent spectra taken from a microdroplet exhibiting volume stabilization. At acquisition 13, evaporation rate equals the condensation rate at a stable equilibrium point, initiating the stabilization mechanism. Starting at this point, despite any change in the ambient humidity, the two WGMs stop drifting. The high quality WGMs at around $583.8 \mathrm{~nm}$ and $589.3 \mathrm{~nm}$ remain stable within a resolution limited FWHM of $0.07 \mathrm{~nm}$ until the end of the experimental period $(\sim 79 \mathrm{sec})$. The observed spectral linewidth of $0.07 \mathrm{~nm}$ corresponds to a volume stabilization within $0.42 \mathrm{fl}$ (radius stabilization of $0.8 \mathrm{~nm}$ ).

By blocking the laser for short time intervals, the stable volume of a microdroplet could be changed stepwise. This is demonstrated in Fig. 4c where laser beam is blocked for $500 \mathrm{~ms}$ before acquisition 10. The interim laser blocking causes the WGMs to start drifting to larger wavelengths. The microdroplet's volume then stabilizes at a consecutive stable equilibrium point starting from acquisition 26.

\subsection{Observation of lasing in Rhodamine B doped microdroplets}

Because they confine high quality optical resonances in small volumes; optical microcavities [1] are attractive in developing ultralow threshold lasers. Such light sources hold a great promise for applications in optical communications systems and fundamental studies in cavity quantum electrodynamics. Up to date laser emission has been observed from various different optical microcavities. Examples include microdisks [21], microspheres [22], micropillars [23], and photonic crystal defect microcavities [24]. 
Experiments performed more than 20 years ago demonstrated laser emission from liquid microdroplets flying in air $[14,25,26]$. In these experiments, a stream of dye-doped ethanol droplets was excited by a pulsed laser. Laser action was observed by analyzing the emission spectra collected from single microdroplets excited by single pump pulses. Recently lasing was also observed from levitated, Rhodamine 6G doped droplets [27]. Here, we present our experiments showing laser emission from single, stationary, dye-doped glycerol/water microdroplets located on a superhydrophobic surface [6].
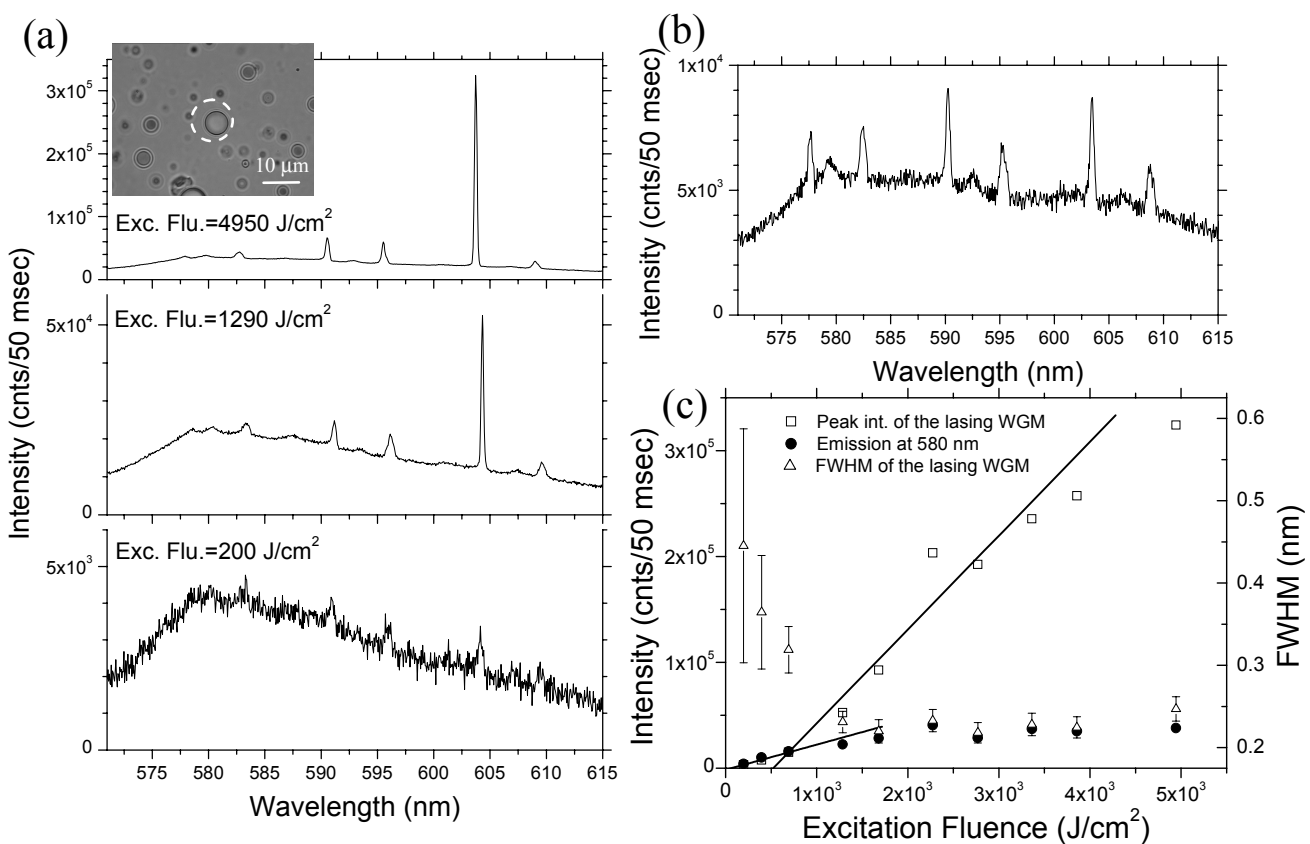

Fig. 5. (a) Fluorescence spectra obtained from a $7 \mu \mathrm{m}$ diameter microdroplet for three different excitation fluences. Inset: Optical microscope image of the microdroplet, dashed circle shows the $12 \mu \mathrm{m}$ diameter area illuminated by the excitation laser. (b) Fluorescence spectrum recorded after a total irradiation time of $1 \mathrm{sec}$ following the spectra shown in (a), under a constant excitation fluence of 4950 $\mathrm{J} / \mathrm{cm}^{2}$. (c) Excitation fluence dependence of the peak intensity of the lasing WGM and its FWHM.

Figure 1a shows the power dependent fluorescence spectra obtained from an $7 \mu \mathrm{m}$ diameter microdroplet exhibiting laser emission. In Fig. 1a for an excitation fluence of 200 $\mathrm{J} / \mathrm{cm}^{2}$, the intensity of the WGM at around $604 \mathrm{~nm}$ is comparable to the intensity of other WGMs and background Rhodamine B emission. At higher excitation fluences, this WGM dominates the emission spectrum. The nonlinear power dependence shown in Fig. $1 \mathrm{~b}$ is an indication of laser emission. The observed nonlinear power dependence is an indication of laser emission. The lasing WGM is spectrally located in the red-shifted portion of the Rhodamine B emission spectrum due to the overlap of the absorption and emission bands on the blue end of the dye emission spectrum, favoring lasing at red-shifted wavelengths. This is consistent with the previous demonstrations using microdroplets flying in air. Due to the errors caused by the deviations from the ideal spherical geometry of the stationary microdroplets, no attempt was made to exactly identify the WGMs seen in Fig. 1. It is speculated that the lasing WGM has a mode order of 1 because of its relatively high quality factor. Laser emission was observed to decay with photobleaching. Fig. 1b shows the emission spectrum recorded after a total irradiation time of $1 \mathrm{sec}$ following the spectra shown 
in Fig. 1a, under a constant excitation fluence of $4950 \mathrm{~J} / \mathrm{cm}^{2}$. As can be seen in Fig. 1b, the intensity of the WGM at around $604 \mathrm{~nm}$ becomes comparable to the intensities of the other WGMs as a result of photobleaching, and lasing is no longer observed in this case. In Fig. 1a, the free spectral range (FSR) of the lasing WGM is measured to be $13.1 \mathrm{~nm}$, corresponding to an ideal glycerol microsphere (refractive index $\mathrm{n}=1.47$ ) diameter of $7.5 \mu \mathrm{m}$. This fits fairly well with the $7 \mu \mathrm{m}$ diameter measured from the microscope image for this microdroplet. The difference between the theoretical estimate and the experimental result is explained by the uncertainty in the diameter measurement using the microscope image.

Power dependent intensities of the lasing WGM as well as the background emission at $580 \mathrm{~nm}$ are plotted in Fig. 1c. The background emission at $580 \mathrm{~nm}$ shows linear power dependences at low excitation powers. Above an excitation fluence of $2800 \mathrm{~J} / \mathrm{cm}^{2}$, it becomes saturated, without any clear indication of nonlinear power dependence. On the other hand, the lasing WGM exhibits nonlinear power dependence above a certain threshold excitation fluence. The threshold fluence was estimated from the crossing point of the linear least squares fits to the power-dependent emission data, and was determined to be $690 \mathrm{~J} / \mathrm{cm}^{2}$.

Another indication of laser emission is the decrease in the spectral width of the lasing mode. This was observed for the specific lasing WGM shown in Fig. 1. The full-width-athalf-maximum (FWHM) of the lasing WGM was measured to decrease from $0.45 \mathrm{~nm}$ at the excitation fluence of $200 \mathrm{~J} / \mathrm{cm}^{2}$ to a resolution-limited width of $0.25 \mathrm{~nm}$ at excitation powers exceeding $1290 \mathrm{~J} / \mathrm{cm}^{2}$, as shown in Fig. 1c.

\section{CONCLUSION}

In this article we have reviewed our work introducing a microdroplet of a hydrophilic liquid resting on a superhydrophobic surface as a novel optical microcavity. Largely tunable resonances were demonstrated by using evaporation/condensation in a humidity chamber. The observed large tenability can lead to applications in optical communication systems. For future applications, faster tuning methods can be developed with an applied external force or electric field [28]. Our experiments also revealed an intriguing high resolution volume stabilization mechanism for dye-doped water microdroplets. The mechanism relied on the interplay between the constant condensation rate and size dependent laser induced heating.

Laser emission was also presented as a result of pumping dye-doped glycerol/water microdroplets with a pulsed laser. As optical microcavities exhibiting laser emission, microdroplets standing on a superhydrophobic surface can be attractive alternatives to solid optical microcavities standing on substrates, e.g. microdisks, micropillars, and photonic crystal defect microcavities. Microdroplets standing on a superhydrophobic surface do not pose any microfabrication challenges. They also bring together the advantage of easy deformability. This can lead to the realizations of tunable microcavity lasers by using evaporation/condensation, or electric field tuning [28].

Besides, enhancement of optical field intensities in the glycerol/water microdroplet might lead to applications in fluid sensing or precise measurements of optical interactions in minute samples of biological specimens or biomolecules. The results presented here can also find applications in characterizing superhydrophobice surfaces and investigating the dynamics at liquid/solid interfaces due to the high sensitivity to the size and shape of the microdroplets. 


\section{Acknowledgments}

This work was supported by the Scientific and Technological Research Council of Turkey (Grant No. TÜBİTAK-105T500). The authors thank the Alexander von Humboldt Foundation for equipment donation. A. Kiraz acknowledges the financial support of the Turkish Academy of Sciences in the framework of the Young Scientist Award program (Grant No. A.K/TÜBAGEBIP/2006-19).

\section{References}

[1] K. J. Vahala, "Optical microcavities," Nature 424, 839-846 (2003) [doi:10.1038/nature01939].

[2] S. Arnold, S. Holler, J. H. Li, A. Serpengüzel, and W. F. Auffermann, "Aerosol particle microphotography and glare-spot absorption spectroscopy," Opt. Lett. 20, 773-775 (1995).

[3] R. J. Hopkins, L. Mitchem, A. D. Wardw, and J. P. Reid, "Control and characterisation of a single aerosol droplet in a single-beam gradient-force optical trap," Phys. Chem. Chem. Phys. 6, 4924-4927 (2004) [doi:10.1039/b414459g].

[4] A. Kiraz, A. Kurt, M. A. Dündar, and A. L. Demirel, "Simple largely tunable optical microcavity," Appl. Phys. Lett. 89, 081118 (2006) [doi:10.1063/1.2335371].

[5] A. Kiraz, A. Kurt, M. A. Dündar, M. Y. Yüce, and A. L. Demirel, "A method for volume stabilization of single, dye-doped water microdroplets with femtoliter resolution," J. Opt. Soc. Am. B 24, 1824-1828 (2007) [doi:10.1364/JOSAB.24.001824].

[6] A. Kiraz, A. Sennaroglu, S. Doganay, M. A. Dündar, A. Kurt, H. Kalaycýoðlu, and A. L. Demirel, "Lasing from single, stationary, dye-doped glycerol/water microdroplets located on a superhydrophobic surface," Opt. Commun. 276, 145-148 (2007) [doi:10.1016/j.optcom.2007.04.026].

[7] M. Y. Yüce, A. L. Demirel, and F. Menzel, "Tuning the surface hydrophobicity of polymer/nanoparticle composite films in the Wenzel regime by composition," Langmuir 21, 5073-5078 (2005) [doi:10.1021/la050033y].

[8] S. C. Hagness, D. Rafizadeh, S. T. Ho, and A. Taflove, "FDTD microcavity simulations: design and experimental realization of waveguide-coupled single-mode ring and whispering-gallery-mode disk resonators," J. Lightwave Technol. 15, 21542164 (1997) [doi:10.1109/50.641537].

[9] P. Heimala, P. Katila, J. Aarnio, and A. Heinamaki, "Thermally Tunable Integrated Optical Ring Resonator with Poly-Si Thermistor," J. Lightwave Technol. 14, 22602267 (1996) [doi:10.1109/50.541217].

[10] D. Armani, B. Min, A. Martin, and K. J. Vahala, "Electrical thermo-optic tuning of ultrahigh-Q microtoroid resonators," Appl. Phys. Lett. 85, 5439-5441 (2004) [doi:10.1063/1.1825069].

[11] B. Maune, R. Lawson, C. Gunn, A. Scherer, and L. Dalton, "Electrically tunable ring resonators incorporating nematic liquid crystals as cladding layers," Appl. Phys. Lett. 83, 4689-4691 (2003) [doi:10.1063/1.1630370].

[12] W. V. Klitzing, R. Long, V. S. Ilchenko, J. Hare, and V. Lefevre-Seguin, "Tunable whispering gallery modes for spectroscopy and CQED experiments," New J. Phys. 3, 1411-1414 (2001) [doi:10.1088/1367-2630/3/1/314].

[13] S. Kuiper, and B. W. Hendricks, "Variable-focus liquid lens for miniature cameras," Appl. Phys. Lett. 85, 1128-1130 (2004) [doi:10.1063/1.1779954].

[14] S.-X. Qian, J. B. Snow, H. M. Tzeng, and R. K. Chang, "Lasing droplets: highlighting the liquid-air interface by laser emission," Science 231, 486-488 (1986) [doi:10.1126/science.231.4737.486]. 
[15] R. J. Hopkins, R. Symes, R. M. Sayer, and J. P. Reid, "Determination of the size and composition of multicomponent ethanol/water droplets by cavity-enchanced Raman scattering," Chem. Phys. Lett. 380, 665-672 (2003) [doi:10.1016/j.cplett.2003.09.048].

[16] J. P. Barton, D. R. Alexander, and S. A. Schaub, "Internal and near-surface electromagnetic fields for a spherical particle irradiated by a focused laser beam," $J$. Appl. Phys. 64, 1632-1639 (1988) [doi:10.1063/1.341811].

[17] J. P. Barton, D. R. Alexander, and S. A. Schaub, "Internal fields of a spherical particle illuminated by a tightly focused laser beam: Focal point positioning effects at resonance," J. Appl. Phys. 65, 2900-2906 (1989) [doi:10.1063/1.342736].

[18] J. D. Eversole, H.-B. Lin, and A. J. Campillo, "Input/output resonance correlation in laser-induced emission from microdroplets," J. Opt. Soc. Am. B 12, 287-296 (1995).

[19] G. Gouesbet, B. Maheu, and G. Grehan, "Light scattering from a sphere arbitrarily located in a Gaussian beam, using a Bromwich formulation," J. Opt. Soc. Am. A 5, 1427-1443 (1988).

[20] J. A. Lock, "Improved Gaussian beam-scattering algorithm," Appl. Opt. 34, 559-570 (1995).

[21] S. L. McCall, A. F. J. Levi, R. E. Slusher, S. J. Pearton, and R. A. Logan, "Whispering-gallery mode microdisk lasers," Appl. Phys. Lett. 60, 289 (1992) [doi:10.1063/1.106688].

[22] V. Sandoghdar, F. Treussart, J. Hare, V. Lefevre-Seguin, J.-M. Raimond, and S. Haroche, "Very low threshold whispering-gallery-mode microsphere laser," Phys. Rev. A 54, R1777 (1996) [doi:10.1103/PhysRevA.54.R1777].

[23] J. L. Jewell, S. L. McCall, Y. H. Lee, A. Scherer, A. C. Gossard, and J. H. English, "Lasing characteristics of GaAs microresonators," Appl. Phys. Lett. 54, 1400 (1989) [doi:10.1063/1.100679].

[24] O. Painter, R. K. Lee, A. Scherer, A. Yariv, J. D. O'Brien, P. D. Dapkus, and I. Kim, "Two-dimensional photonic band-gap defect mode laser," Science 284(5421), 18191821 (1999) [doi: 10.1126/science.284.5421.1819].

[25] H. B. Lin, J. D. Eversole, and A. J. Campillo, "Spectral properties of lasing microdroplets," J. Opt. Soc. Am. B 9, 43-50 (1992).

[26] H.-M. Tzeng, K. F. Wall, M. B. Long, and R. K. Chang, "Laser emission from individual droplets at wavelengths corresponding to morphology-dependent resonances," Opt. Lett. 9, 499-501 (1984).

[27] H. Azzouz, L. Alkhafadiji, S. Balslev, J. Johansson, N. A. Mortensen, S. Nilsson, and A. Kristensen, "Levitated droplet dye laser," Opt. Exp. 14, 4374-4379 (2006) [doi:10.1364/OE.14.004374].

[28] V. H. Kwong, A. M. Mossman, and A. L. Whitehead, "Control of reflectance of liquid droplets by means of electrowetting," Appl. Opt. 43, 808 (2004) [doi:10.1364/AO.43.000808].

\begin{abstract}
Alper Kiraz is an assistant professor of physics at Koç University. He received his BS degree in electrical-electronics engineering from Bilkent University in 1998, MS and $\mathrm{PhD}$ degrees in electrical and computer engineering from the University of California, Santa Barbara in 2000 and 2002, respectively. He is the author of more than 20 journal papers and has been an author of one book chapter. His current research interests include optical microcavities, single molecule microscopy, and optical tweezers. He is a member of OSA (Optical Society of America), and DPG (Deutsche Physikalische Gesellschaft).
\end{abstract}

Mehmet A. Dündar: biography not available. 
Adem L. Demirel is associate professor of Chemistry at Koc University, Istanbul, Turkey. $\mathrm{He}$ received his $\mathrm{Ph} . \mathrm{D}$. degree in physics from the University of Illinois, Urbana-Champaign, USA in 1996. His current research interests include functional surfaces and self-organizaton of polymers.biography not available.

Sultan Doğanay: biography not available.

Adnan Kurt received his B.S. (Electrical Engineering) and M.S. (Physics) degrees from Boğaziçi University, Istanbul, Turkey in 1984 and 1987, respectively. Between 1985 and 1990, he was a Research Assistant in the Departments of Physics and Psychology at Bogazici University, and worked on laser speckle, pulsed gas laser design, neuro-computing, and animal learning. Later, he joined the research group at the Center for Electroneurophysiology at Istanbul University Medical School, and continued work in neurophysiology and human brain studies (1990-1995). He also worked for Mitra Inc., and Teknofil Ltd., doing business in printing, imaging, electrophotography, and instrumentation control. In 1999, he joined the Department of Physics at Koç University as a Research Engineer. His current research interests include solid-state laser design, optical and electronic instrumentation, and laser spectroscopy.

Alphan Sennaroglu received his BS, MS, and PhD degrees in Electrical Engineering from Cornell University in 1988, 1990, and 1994, respectively. After joining Koç University in 1994, he established the Laser Research Laboratory. Currently, he is a professor in the Department of Physics. Alphan Sennaroglu's research interests include solid-state lasers, ultrafast optics, spectroscopy of novel laser and amplifier media, and nonlinear optics. He was a visiting researcher at the Massachusetts Institute of Technology during the 2002-2003 academic year and in the Summers of 2005 and 2006. He served as the program chair for the Solid-State Lasers and Amplifiers Conference that was held in 2004 and 2006 during the Photonics Europe meeting in Strasbourg, France. Alphan Sennaroglu is an associate member of the Turkish Academy of Sciences (TUBA), a Senior Member of IEEE (Institute of Electrical and Electronics Engineers) and a member of OSA (Optical Society of America), SPIE (The International Society for Optical Engineering), Optics Committee of Turkey, Tau Beta Pi, and Eta Kappa Nu. In 1999, he founded the Leos Turkish Chapter of the IEEE Lasers and Electro-Optics Society and served as the Chapter president between 1999 and 2003. He received the 2002 ICTP/ICO Award (ICTP: International Center for Theoretical Physics, ICO: International Commission for Optics), 2001 Werner-von-Siemens Award (Koç University), 2001 TUBA Young Scientist Award, 1998 Tubitak (Scientific and Technical Research Council of Turkey) Young Scientist Award, Sage Graduate Fellowship (Cornell University, 1989-1990), Sibley Prize of Electrical Engineering (Cornell University, 1988), and Amideast undergraduate scholarship (1984-1988).

M. Yavuz Yüce is currently a PhD student at Konstanz University, Germany, in the research group of Prof. Andreas Zumbusch. He received his BS degrees in mechanical engineering and in physics, and his MS degree in materials science and engineering from Koc University, Turkey in 2004 and 2006 respectively. His prospective research will be on investigation of physical phenomena in thin polymer films using single molecule spectroscopy and microscopy. 